Methods We pooled data from all Meloxicam clinical trials that were at least 3 weeks in duration and included at least 20 patients per arm. Thirty-five trials with 27,039 patients on Meloxicam, comparator NSAID?s, or placebo were included in the analysis. Nearly $39 \%(8109)$ patients were over age 65 . Since trials were of varying durations, we report event rates as incidence per 100 patient-years of exposure.

Results Of the 27,039 patients studied, 15,071 received meloxicam in doses of 7.5 ? $30 \mathrm{mg}$ daily. A total of 11,078 patients took diclofenac, naproxen or piroxicam. An additional 736 patients were randomised to placebo. The number of patients with cardiovascular and renal events adverse events is shown in the Table 1 below. Incidence rates in parenthesis refer to rates per 100 patient years.

\begin{tabular}{llll}
\multicolumn{4}{l}{ Abstract THU0258 Table 1} \\
\hline & Meloxicam & NSAIDs & Placebo \\
\hline Intent-to-treat patients & $(\mathrm{n}=15,071)$ & $(\mathrm{n}=11,078)$ & $(\mathrm{n}=736)$ \\
$\begin{array}{l}\text { Patient Years } \\
\text { Events }\end{array}$ & 3129 & 1202 & 113 \\
Myocardial Infarction & $18(0.58)$ & $8(0.67)$ & $2(1.8)$ \\
Cardiac Failure & $15(0.48)$ & $7(0.58)$ & $0(0)$ \\
Oedema, Peripheral & $98(3.13)$ & $79(6.57)$ & $1(0.88)$ \\
Hypertension & $82(2.62)$ & $32(2.66)$ & $5(4.42)$ \\
Hypertension, Aggravated & $25(0.8)$ & $15(1.25)$ & $2(1.77)$ \\
\hline Numbers in parenthesis reflect incidence rates per 100 patient years. &
\end{tabular}

Conclusion Meloxicam in doses of 7.5-30 mg is not associated with increased incidence of cardiovascular or renal toxicity.

\section{THU0259 THE RISK FACTORS FOR PAIN IN LOWER EXTREMITIES JOINTS IN UNIVERSITY STUDENTS OF PHYSICAL CULTURE}

J Flicinski, M Brzosko, K Prajs, H Przepiera-Bedzak. Rheumatology, Pomeranian Academy of Medicine, Szzzecin, Poland

\subsection{6/annrheumdis-2001.803}

Background Musculoskeletal pain is a very common complaint. Its prevalence increases with age but it is experienced also by young individuals. In a previous study concerning prevalence of musculoskeletal pain in joints of upper and lower extremities among university students of physical culture it was found that it occurred within the year in $45,9 \%$ of the whole group. Students complained of pain the most frequently in lower extremities joints $(35,0 \%)$ while pain in upper extremities joints was reported by $20,87 \%$ of them. This study is a further step to find the most important factors influencing the occurrence of pain in lower extremities joints in this group.

Objectives To determine the risk factors of the occurrence of pain in lower extremities joints in university students of physical culture.

Methods The study was performed on 503 university students of physical culture, mean age 22,6 yrs (20-28 yrs), 218 women and 285 men. The subjects received a questionnaire concerning questions about musculoskeletal disorders in last 12 months. A reported joint pain had to last for at least $24 \mathrm{~h}$ to be considered as relevant. The questionnaire included also question about psycho-social factors, families, smoking habits and many other aspects of everyday life. The questionnaire was responded by
$95,8 \%$ of students. The potential relationship of the investigated 27 variables was assessed in multivariate logistic regression model and then the role of significant variables was counted as relative risk with $95 \%$ of confidence interval in univariate logistic regression model. For statistical evaluation of the data STATA 5.0 software was used.

Results Multivariate logistic regression has revealed that in women only stress related to personal problems (OR 2,3; CI 1,3-4,1), treatment for any long-lasting disease (OR 6,12; CI 1,9-20,1), frequent headaches (OR 2,1; CI 1,1-3,7) were significantly related to reporting pain in lower extremities joints.

In male students reporting joint pain in lower extremities was significantly associated with body height (there was less pain for tall subjects, OR 0,4; CI 0,2-0,8), conflict with lecturers or teachers (OR 2,0; CI 1,2-3,5) and also with bringing up children (OR 3,0; CI 0,9-9,8). In both male and female students there were other risk factors of back pain like abdominal pain, conflicts with fellow students or smoking however the were not independent ones in multivariate logistic regression.

Conclusion In female and male students pain in lower extremities joints was associated with some other health problems or with stress. In males also bringing up children influenced significantly occurrence of pain in lower extremities joints.

\section{THU0260 RHEUMATIC DISEASES AS A REASON OF PHYSICIAN OFFICE VISITS (POV) COMPARED TO OTHER MAJOR DISEASE GROUPS (MDG) IN URBAN, SUBURBAN AND RURAL GREEK POPULATION}

S Aslanides, A Andrianakos, E Kaskani, Z Nikolia, E Vafiadou, K Pantelidou, G Kaziolas, P Krachtis, L Kontelis, D Karamitsos, F Christogiannis, P Dandis. Hellenic Foundation for Rheumatological Research, Hellenic Foundation for Rheumatological Research, Athens, Greece

\subsection{6/annrheumdis-2001.804}

\section{Background}

Objectives The estimation of patient POV rate because of RD or any other MDG was one of the aims of a population-based epidemiological study of RD in Greece (ESORDIG study).

Methods This community based study was conducted from 1996 to 1999 (3 years) in two urban (U), one suburban (SU) and four rural $(\mathrm{R})$ communities of Greece. All adult ( $>18$ years) residents were included (8,547 subjects), along with 2,100 randomly selected subjects of $1 \mathrm{SU}(1: 3)$ and $1 \mathrm{R}(1: 2)$ communities. The study was performed by sixteen rheumatologists; 8,740 subjects were included (out of a total of 10,647 , response rate $82.1 \%$ ). The study included a detailed interview based on a standardised questionnaire. Established classification criteria (ECC) were used for the diagnosis of RD, while, for the purpose of the study, such criteria were set for RD without ECC. The questionnaire included distinct questions about POV due to any disorder two weeks prior to the interview.

Results The POV rate for RD was $2.7 \%$ in the general population (GP), higher in women (3.1\%) than in men $(2.2 \%, \mathrm{p}<$ $0.005)$; the highest POV rate was observed in the 49-58 yeargroup. The overall POV rate due to any disorder was $13.3 \%$ in GP; RD were the most common reason (19.9\%), followed by cardiovascular diseases (CVD, 19.1\%), respiratory diseases (ReD, 13.6\%), and endocrine-metabolic diseases (12.5\%). Analyses for age and sex showed that in the 29-58 year-groups RD were the most common reason for POV (26.3\%), followed by ReD (11.5\%), while in the $>58$ year-groups CVD were the most 
common (29.1\% of POV), followed by RD (16.1\%). No differences were observed between communities.

Conclusion These findings show that $\mathrm{RD}$ are the most common reason for POV ( 1 in 5 visits), mainly in the productively active age groups.

\section{Education}

\section{SAT0225 A NOVEL WEB BASED RHEUMATOLOGY EDUCATIONAL RESOURCE - INTERACTIVE COMPUTERISED CASE STUDIES FOR UNDERGRADUATE TEACHING}

${ }^{1}$ AS Wilson, ${ }^{2} \mathrm{GD}$ Kitas, ${ }^{1} \mathrm{SJ}$ Bowman, ${ }^{1} \mathrm{P}$ Llewellyn, ${ }^{1} \mathrm{PA}$ Bacon, ${ }^{1} \mathrm{SP}$ Young. ${ }^{1}$ Rheumatology, University of Birmingham, Birmingham, UK; ${ }^{2}$ Rheumatology, The Guest Hospital, Birmingham, UK

\subsection{6/annrheumdis-2001.805}

Background We have applied the principal that the undergraduate curriculum should have a reduced burden of factual information and learning through curiosity should be encouraged, ${ }^{1}$ to the education of our rheumatology students.

Objectives We have applied advances in computer technology to develop a web based educational strategy and evaluated its use from the perspective of the student/user.

Methods A teaching needs assessment conducted on a cohort of 30 students prompted the development of a novel interactive case studies programme, accessible through the internet. This programme utilises active server page technology (TM) reading from a database of case study information (anonymised). The user can select predefined questions, perform a virtual physical examination and gain diagnostic information by requesting laboratory tests. The user is then required to make a diagnosis and select an appropriate treatment regimen for that patient. To introduce realism, performance is evaluated by an indication of the patients outcome and the financial expenditure incurred for their consultation. Usage data was collected from online questionnaire and by computer generated log file analysis.

Results All students agreed that computer based learning was a useful adjunct to traditional teaching and greater than 95\% expressed strong support for the use of computerised case studies. All questionnaire respondents $(n=17)$ agreed that the computer programme was well organised, realistic and that they had learnt from the experience. Although this teaching aid is primarily aimed at our local teaching hospitals, log file analysis indicates that there have been 239 visits to the site (181 unique) from North America, Europe, Asia and Oceania, suggesting a widespread interest.

Conclusion This novel interactive web based programme, based on real life patient information, will serve as a teaching resource for our allied hospitals and introduce some variety into more formal teaching practices by promoting self-learning. The techniques employed could be adaptable to other situations which would benefit from increased user interaction and in a dispersed learning environment.

\section{REFERENCE}

1 General Medical Council report "Tomorrow's Doctors", 1993

\section{SAT0226 A REVIEW OF AN INTERNET BASED 'ARTHRITISHELP' RESOURCE: (HTTP://RHEUMA. BHAM. AC. UK)}

${ }^{1}$ AS Wilson, ${ }^{2} \mathrm{GD}$ Kitas, ${ }^{1} \mathrm{I}$ Lal, ${ }^{1} \mathrm{P}$ Llewellyn, ${ }^{1} \mathrm{PA}$ Bacon, ${ }^{1} \mathrm{SP}$ Young. ${ }^{1}$ Rheumatology, University of Birmingham, Birmingham, UK; ${ }^{2}$ Rheumatology, The Guest Hospital, Dudley, UK

10.1136/annrheumdis-2001.806

Background The Internet is an important way of delivering medical information to assist in improving patient's self-management of their disease.

Objectives We have established an Arthritis education website ('Arthritis Help') and investigated its use over two years.

Methods Computer generated log file analysis and online questionnaire were used to create usage profiles of our web site.

Results An average of 288 people visited our site each day predominantly, but not exclusively, from America and the UK (49\% of users). The typical questionnaire respondent $(n=770)$ was a $30+$ year old American female with arthritis, accessing the Internet from home. They had previously obtained information from medical staff or in written form but were now more likely to use the Internet. 167 out of 585 respondents found our site to be useful prompting them to seek more information (29\%), change their behaviour or engage in more effective discussions with their physician (15\%). 427 people responded to our comments section of the questionnaire of which 333 made clear suggestions. $21 \%$ of these respondents were enquiring about a specific medical condition or requesting more information on that condition. 20\% wanted more detailed information about rheumatoid conditions, treatments or drug information. 9\% wished to see more information on 'alternative treatments' whilst diet, exercise, new treatments, a questions and answers section, a section to air shared experiences and links to other information formed $5-6 \%$ of respondents suggestions.

Conclusion This data indicates that it is possible to use the internet to deliver medical information to it's target audience and that this process can have some impact on the way disease is self-managed. This information may aid more focused web-site design to maximise use and the potential benefits from such a resource.

\section{SAT0227 AN ACADEMIC ARTHRITIS WEBSITE IS MOST FREQUENTLY UTILISED FOR ARTHRITIS NEWS AND TREATMENT INFORMATION}

JM White, JM Bathon, AK Matsumoto. Medicine, Johns Hopkins University, Baltimore, USA

\subsection{6/annrheumdis-2001.807}

Background Medical and pseudo-medical information sites are the most heavily trafficked sites on the Worldwide Web. The Hopkins Arthritis Web Site was launched in late December 1998 to provide credible information about arthritis and its treatment to health care providers and patients.

Objectives Our goal was to evaluate the topics most frequently accessed by visitors to the Site.

Methods The information data base on the Site consists of a large body of ?static? information, as well as two interactive forums. The number of ?requests? for each page was monitored by Web Trends Enterprise Reporting Server. The number of requests per page was adjusted for number of months that the page was live, and sorted by topic into 1 of 6 categories. Requests were readjusted for number of pages per topic and calculated as a percent of total requests. Data were based on statistics compiled for the year 2000 . 